\title{
Aggregation of Power Losses in Radio Link Budgeting
}

\author{
Patrick O. Olabisi \\ Telecoms Programme, Dept of Electrical \& Computer Engineering, Bells University of Technology, Ota, \\ Nigeria.
}

\begin{abstract}
In the design of wireless communication links between two sites, issues of range, that is, how far the transmitted signal would go given certain transmission parameters, and the throughput of such link, are of great concern to the radio engineer such that several loss factors militating against the strength of the transmitted signal as it travels through the medium to the receiver were given holistic consideration in this work. Major loss factors like antenna feeder loss, antenna mismatch loss, propagation path loss, signal polarization loss, multipath propagation loss, obstruction loss and diffraction loss were discussed. Efforts at reducing the presence and effects of these losses on transmitted signal in a radio link would greatly enhance the efficiency of the transmission system.
\end{abstract}

Keywords: Radio link budgeting, signal power loss, transmitted power, receiver sensitivity, antenna, propagation.

\section{Introduction}

Radio link budgeting is accounting for all power parameters of the signal in a radio link from the transmitter amplifier circuitry to the transmitting antenna, the transmission medium, receiving antenna, and the receiver detection circuitry with respect to the receiver sensitivity relative to the received signal power. In signal power budgeting consideration is given to the transmitted power, power gains and power losses in the link, to be sure that the received power is greater than the receiver sensitivity, so that the communication is sensible and decipherable.

The signal power radiated by the transmitter antenna in a radio communication link suffers various losses from the level of signal strength coming out of the transmitter power amplifier circuitry to the level of signal strength that is received for demodulation and processing by the receiver circuitry. Shown in figure 1 is a typical radio link, the Fresnel zone created within the link and the propagation condition in the link between the transmitter and the receiver [1]. The power losses suffered by the original signal occur both at the transmitter and receiver ends and also within the transmission medium. The losses are actually not so easy to fully predict, capture and analyze, but a lot have been done to take cognizance of them.

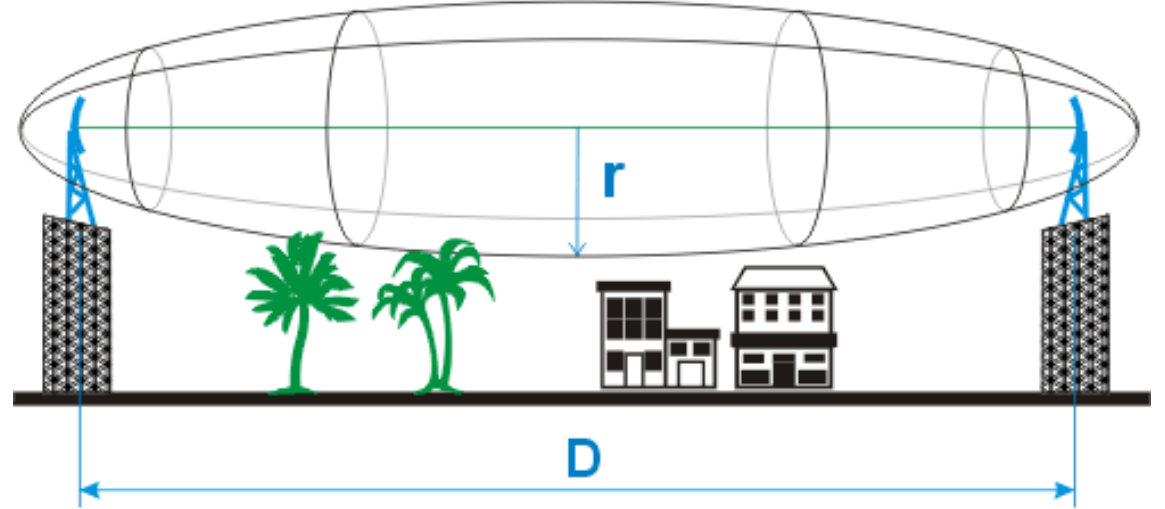

Figure 1: Fresnel Zone and Obstructions in a Radio Link.

This study endeavours by a holistic approach to make an aggregation of losses suffered by the signal power coming out of the transmitter power amplifier through to that entering the receiver for detection. Section II highlights areas of the link where these losses occur, while section III discussed the respective power losses, section IV accounts for the power losses in a radio link budget. Section V concludes the work.

\section{Power Losses in a Radio Link}

Power losses occur in the transmitter and receiver feeder units, in the transmitter and receiver antenna coupling as antenna mismatch loss, in the transmission medium in various forms generally as propagation path 
losses. The propagation path losses comprise of the free space path loss which occurs as a result of the characteristics of the air medium, signal polarization, multipath propagation, obstruction on the propagation path, atmospheric characteristics, obstacles penetration, and pointing errors particularly more pronounced in satellite communication links.

Figure 2 is a simplified diagram of a communication link showing the signal components and the signal paths between the transmitter and the receiver. Shown also are the components wherein the transmitter signal is subjected to power losses. This study in a holistic approach identifies and describes the total power losses suffered by the communication signal. Efforts at identifying and capturing these losses have been discussed severally in lots of literatures over time [2], [3], [4], [5], [6], but in a scattered non-holistic approach, as this study has done. The various power losses are discussed in section III.

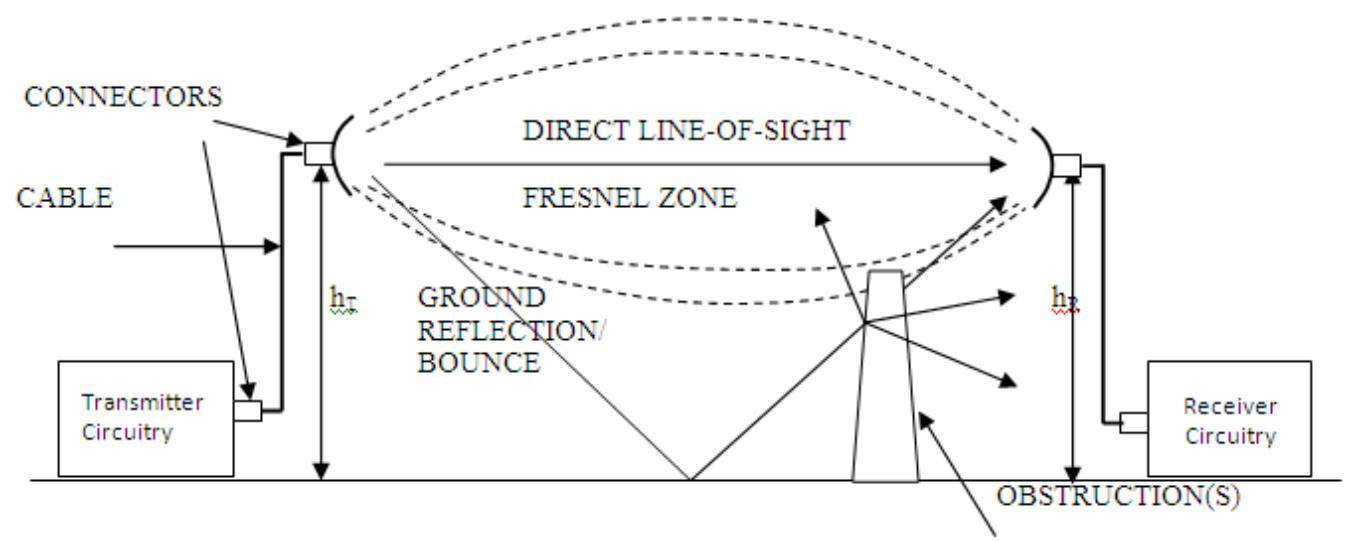

Figure 2: Propagation in a Communication Link.

ITU-R P.530-15 Recommendations [7] indicates that power losses which occur in signal propagation on a terrestrial line-of-sight path relative to the free-space loss (also see Recommendation ITU-R P.525) as being the sum of different contributions given as follows:

- attenuation due to atmospheric gases,

- diffraction fading due to obstruction or partial obstruction of the path,

- fading due to multipath, beam spreading and scintillation,

- attenuation due to variation of the angle-of-arrival/launch,

- attenuation due to precipitation,

- attenuation due to sand and dust storms.

It also indicated that each of these propagation loss contributions has its own characteristics as a function of frequency, path length and geographic location.

\section{Power Losses in a Transmission Link}

Radio signal power losses occur in different types and forms, which include antenna feeder loss, antenna impedance mismatch loss, polarization mismatch loss, path losses, multipath fading losses, obstruction loss, diffraction loss, scattering loss, pointing loss, penetration loss, atmospheric loss, and so on. In this study, the major identifiable power losses in a radio transmission link are discussed in the following sub-sections, starting from losses incurred in feeding the signal from the transmitter's amplifier circuitry to the transmitting antenna, to loss in antenna matching and losses in the transmission medium.

\subsection{Antenna Feeder Losses}

These are losses associated with the antenna connectors, couplers and the signal cable used in connecting the transmitter or receiver circuit to its antenna. Shown in figure $3[8]$ is a typical connection of an antenna feeder system to a base station situated indoor.

The feeder loss include losses from all devices from the base station coupler or port to the indoor jumper, coupling to the main transmission feeder cable, combiners, splitters the outdoor jumper and the coupling to the antenna itself. If the base station radio unit is installed on a rooftop for instance, then we would only be required to bother about loss due to the outdoor jumper since other feeder lines/components would not be necessary.

$\mathrm{RF}$ feeders which are either coax or coaxial feeder, open wire or twin feeder, or waveguide, have been found to possess certain advantages and application effects at different frequency levels [9]. The coaxial feeder which is mostly used particularly in the VHF and UHF frequency ranges, is easy and convenient to install, and 
also cheaper than the waveguide. The waveguide is mostly used in the higher (microwave) frequencies, and has the advantage of low loss than the coaxial at such frequency levels.

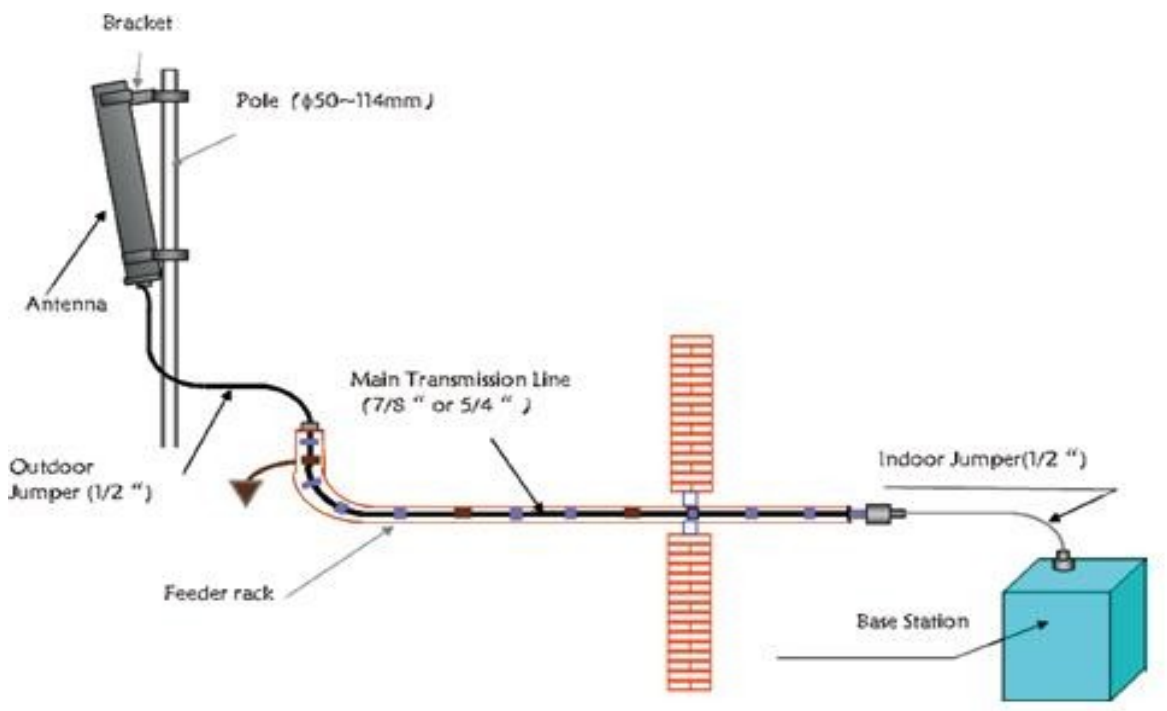

Figure 3: Typical Connection of the antenna feeder system in an indoor base station.

Calculating antenna feeder loss is based on the feeder type and the feeder length, and obtained with the following formula [8]:

Feeder loss $(\mathrm{dB})=$ Feeder loss per $100 \mathrm{~m}(\mathrm{~dB} / 100 \mathrm{~m}) \times$ feeder length $(\mathrm{m}) / 100$.

This feeder loss is noted to be related to the frequency level of the transmitted signal.

\subsection{Antenna Mismatch Loss}

This is power loss due to mismatch between the impedance of the transmitter or receiver circuit and the impedance of the antenna. Here some impedance matching (passive) components may be required to be connected to the transmitter to enhance impedance matching.

To determine if a feeder line and an antenna are properly matched, a Standing Wave Ratio (SWR) meter or a Directional Wattmeter is used to take measurements on the line.

Some of the effects of impedance mismatch in a receiver include the following [10]:

- $\quad$ degrades noise figure, gain and dynamic range in the receiver;

- $\quad$ impacts the efficiency, power gain, maximum output power, and linearity of the power amplifier in the receiver;

- $\quad$ in the worst case, the high standing wave amplitude or possible oscillation caused by the impedance mismatch in the power amplifier could damage the amplifier.

Efforts at correcting and preventing these problems include [10]:

- $\quad$ inserting an isolator or a VSWR protection circuitry in-between the amplifier and the antenna, but this method has some attenuation effects thereby reducing system efficiency;

- $\quad$ using dynamic biasing of the power amplifier; or

- $\quad$ using a tunable matching network, which even in worse mismatch condition can still preserves the amplifier performance.

Correcting mismatch using a tunable matching network involves dynamic measurement of the load impedance which can be estimated from measuring voltages at multiple points of the transmission line. A configuration of this measurement and the tunable matching network in a transceiver circuit is shown in figure 4. 


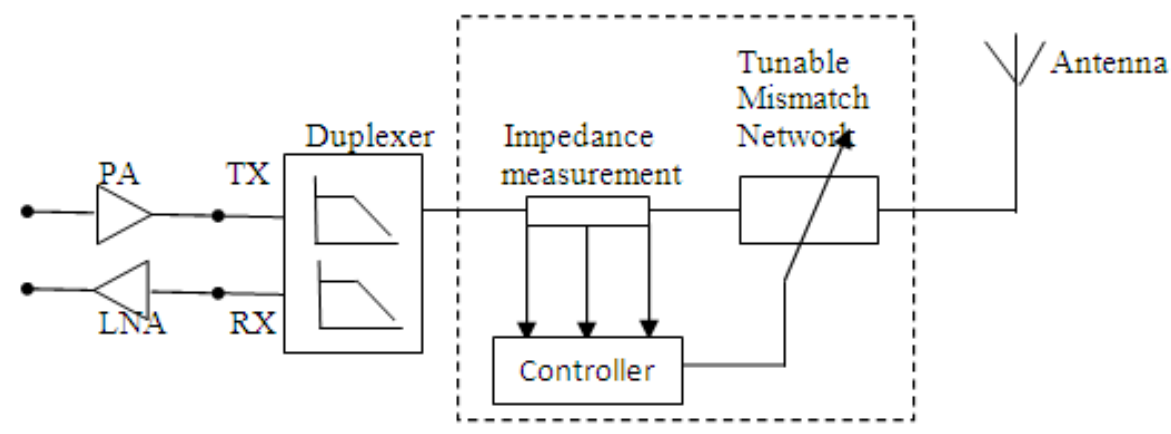

Figure 4: Network connection between antenna and RF circuit.

\subsection{Polarization Mismatch Loss}

Signal polarization is of important consideration in the performance of a radio link with respect to maximum energy transfer between transmitting and receiving antennas. Polarization of antennas, whether linear, circular or elliptic polarization, and whether vertical, horizontal or slant as in the case of linear polarization, is defined by the polarization vector of the radiated signal and can be mismatch when both antennas do not have identical axial ratio, identical polarization sense and the same spatial orientation and where the polarization of each are not of the same orientation [6]. At the receiver, polarization of the antenna is the polarization of the wave incident on the antenna from the direction of the transmitting antenna and determines the maximum power available at the receiving antenna [11]. Un-identically orientation in the antennas polarization orientation, rather known as polarization mismatch, leads to a reduction in energy transfer between them.

For linear polarization, the polarization mismatch loss is given by:

$\Gamma=\cos (\theta)$

where $\theta$ is the angle between polarization vectors.

$\Gamma($ in $\mathrm{dB})=20 \log _{10}(\cos \theta)$

If the difference in alignment angle between the two antennas is $15^{\circ}$ the loss is approximately $0.3 \mathrm{~dB}$, for $30^{\circ}$ we lose $1.25 \mathrm{~dB}$, for $45^{\circ}$ we lose $3 \mathrm{~dB}$ and for $90^{\circ}$ we have an infinite loss. This implies that the greater the mismatch in polarization between a transmitting and receiving antenna, the greater the apparent loss, and polarization loss is any value between zero and infinity. This fact is further analyzed and plotted on table 1[12].

Table 1: Transmitter and Receiver Alignment and Polarization Loss.

\begin{tabular}{|c|c|c|c|c|c|c|c|}
\hline \multirow[t]{3}{*}{$\begin{array}{l}\text { Transmit } \\
\text { Polarization }\end{array}$} & \multirow[t]{3}{*}{$\begin{array}{ll}\text { Receive } & \text { Antenna } \\
\text { Polarization } & \end{array}$} & \multicolumn{6}{|c|}{$\begin{array}{l}\text { Polarization Loss as Ratio of Received Power to Maximum } \\
\text { Power }\end{array}$} \\
\hline & & \multicolumn{2}{|c|}{ Theoretical } & \multicolumn{2}{|c|}{ Practical Hom } & \multicolumn{2}{|c|}{ Practical Spiral } \\
\hline & & Ratio & $\begin{array}{l}\text { Ratio in } \\
\text { dB }\end{array}$ & Ratio & $\begin{array}{l}\text { Ratio } \\
\text { in dB }\end{array}$ & Ratio & $\begin{array}{l}\text { Ratio } \\
\text { in dB }\end{array}$ \\
\hline Vertical & Vertical & 1 & 0 & * & $*$ & N/A & $\mathrm{N} / \mathrm{A}$ \\
\hline Vertical & Slant $\left(45^{\circ}\right.$ or $\left.135^{\circ}\right)$ & $1 / 2$ & -3 & $*$ & $*$ & N/A & N/A \\
\hline Vertical & Horizontal & 0 & $-\infty$ & $1 / 100$ & -20 & N/A & N/A \\
\hline Vertical & Circular (RH or LF) & $1 / 2$ & -3 & $*$ & $*$ & $*$ & $*$ \\
\hline Horizontal & Horizontal & 1 & 0 & $*$ & $*$ & N/A & N/A \\
\hline Horizontal & Slant $\left(45^{\circ}\right.$ or $\left.135^{\circ}\right)$ & $1 / 2$ & -3 & $*$ & $*$ & N.A & N.A \\
\hline Horizontal & Circular (RH or LF) & $1 / 2$ & -3 & $*$ & * & $*$ & $*$ \\
\hline Circular (Right-Hand) & Circular (Right-Hand) & 1 & 0 & $*$ & $*$ & $*$ & $*$ \\
\hline Circular (Right-Hand) & Circular (Left-Hand) & 0 & $-\infty$ & $1 / 100$ & -20 & $1 / 10$ & -10 \\
\hline Circular (Right or Left) & Slant $\left(45^{\circ}\right.$ or $\left.135^{\circ}\right)$ & $1 / 2$ & -3 & $*$ & $*$ & $*$ & $*$ \\
\hline
\end{tabular}

- Approximately the same as theoretical.

- Switching transmit and receive antennas will give the same result.

Polarization loss factor (PLF) is stated as the electromagnetic loss of power as a result of polarization mismatch, and it is given by [11]:

$\operatorname{PLF}=\left|\hat{\rho}_{\mathrm{i}} \cdot \hat{\rho}_{\mathrm{A}}\right|^{2}$

where $\hat{\rho}_{\mathrm{i}}$ is polarization vector incident on the antenna. 
When PLF $=\left|\hat{\rho}_{\mathrm{i}} \cdot \hat{\rho}_{\mathrm{A}}\right|^{2}=1$, the antennas are polarization aligned or matched and there is no polarization loss. When PLF $=\left|\hat{\rho}_{\mathrm{i}} \cdot \hat{\rho}_{\mathrm{A}}\right|^{2}=\cos ^{2}(\theta)$, the antennas are misaligned or mismatched and there is remarkable polarization loss.

When PLF $=\left|\hat{\rho}_{\mathrm{i}} \cdot \hat{\rho}_{\mathrm{A}}\right|^{2}=0$, the antennas are orthogonal and the receiving antenna is incapable of receiving the transmitted signal.

The polarization mismatch loss for any angular alignment $\theta$ between major axes is given by [***6]:

$\Gamma(\mathrm{dB})=10 \log \left[\frac{\left(1+\rho_{\mathrm{w}}^{2} \rho_{\mathrm{A}}^{2}+2 \rho_{\mathrm{w}} \rho_{\mathrm{A}} \cos 2 \theta\right)}{\left(1+\rho_{\mathrm{W}}^{2}\right)\left(1+\rho_{\mathrm{A}}^{2}\right)}\right]$

where $\rho_{\mathrm{w}}=\left(\mathrm{r}_{\mathrm{w}}+1\right)\left(\mathrm{r}_{\mathrm{w}}-1\right)=$ the circular polarization ratio of the transmitted wave

$\rho_{\mathrm{A}}=\left(\mathrm{r}_{\mathrm{A}}+1\right)\left(\mathrm{r}_{\mathrm{A}}-1\right)=$ the circular polarization ratio of the receiving antenna

and $\mathrm{r}_{\mathrm{w}}=$ axial ratio of transmitted wave, while $\mathrm{r}_{\mathrm{A}}=$ axial ratio of receiving antenna.

\subsection{Path Losses}

These are power losses due to the attenuation suffered by the signal in the propagation path. The path losses in the air are comprised of two major factors - the Free Space Path Loss (FSPL) and the Flat Earth Path Loss (FEPL). The Free Space Path Loss (FSPL) is obtainable where the antenna heights are located on towers or rooftop of very tall buildings, and where the antenna beams are narrow. The FSPL is given by:

$\mathbf{L}_{\mathbf{F S}}=\left(\frac{4 \pi \mathrm{d}}{\lambda}\right)^{2}=\left(\frac{4 \pi \mathrm{fd}}{\mathrm{c}}\right)^{2}$

$\mathbf{L}_{\mathbf{F S}}(\mathrm{dB})=20 \log _{10}\left(\frac{4 \pi \mathrm{d}}{\lambda}\right)(\mathrm{dB})$

where $\mathrm{d}$ is the distance between the transmitter and the receiver

$\mathrm{f}$ is the frequency of the transmitted signal (in hertz)

$\lambda$ is the wavelength of the transmitted signal (in meters)

$\mathrm{c}$ is the speed of life, $3 \times 10^{8} \mathrm{~m} / \mathrm{s}$

The Flat Earth Path Loss estimates the path loss for links where the antenna heights above ground are usually low like propagations in parking lots, streets, between buildings, within compounds, and so on. The path loss for flat earth propagation is the product of the free space path loss and a ground bounce loss. The FEPL is given by:

$\mathbf{L}_{\mathbf{F E}}=\mathbf{L}_{\mathbf{F S}} \times \mathbf{L}_{\mathbf{G B}}=\left(\frac{4 \pi \mathrm{d}}{\lambda}\right)^{2} \times \frac{1}{2}\left(1+\mathrm{a}^{2}-2 \operatorname{acos}\left(\frac{2 \pi \Delta d}{\lambda}\right)\right)$

where ' $a$ ' is the relative strength of the ground bounce path and it is usually less than or equal to unity (that is, a $\leq 1)$. $\Delta \mathrm{d}$ is the difference in length of the direct path and the ground reflected path, and it is given by:

$\Delta \mathrm{d}=\sqrt{\left(\mathrm{d}^{2}+\left(\mathrm{h}_{1}+\mathrm{h}_{2}\right)^{2}\right)}-\sqrt{\left(\mathrm{d}^{2}+\left(\mathrm{h}_{1}-\mathrm{h}_{2}\right)^{2}\right)}$

Variations in the ground bounce loss, $\mathbf{L}_{\mathbf{G B}}$, are dependent on the size of the distance, $\mathrm{d}$, between transmitter and receiver. At close range, $\mathbf{L}_{\mathbf{G B}}$ is known to vary rapidly with d, particularly for cases where the difference, $\Delta \mathrm{d}$, is greater than or equal to a half wavelength of the signal. At longer range, variation in $\mathbf{L}_{\mathbf{G B}}$ is relative to $\mathrm{d}^{-2}$. With this, the received power decreases as the 4 th power of distance, $\mathrm{d}$.

The free space path loss, from equation (6), is proportional to the square of the distance between the transmitter and the receiver and the frequency of the transmitted signal. The path loss is described by the distancedependent path loss model given by [13]:

$\overline{\mathrm{PL}}(\mathrm{d})[\mathrm{dB}]=\operatorname{PL}\left(\mathrm{d}_{0}\right)+10 \mathrm{n} \log _{10}\left(\frac{\mathrm{d}}{\mathrm{d}_{0}}\right)[\mathrm{dB}]$

where $\overline{\mathrm{PL}}(\mathrm{d})$ is the average path loss value in $\mathrm{dB}$ at a distance, $\mathrm{d}, \mathrm{PL}\left(\mathrm{d}_{0}\right)$ is the path loss in $\mathrm{dB}$ at a reference distance $\mathrm{d}_{0}=1 \mathrm{~m}$, and $\mathrm{n}$ is the path loss exponent which denotes how increase of the path loss with distance, $\mathrm{d}$. for free space propagation, $\mathrm{n}=2$. 


\subsection{Multipath/Fading Losses}

In the link between the transmitter antenna and the receiver antenna, there exist several objects like buildings, trees, and so on, which have different reflective effects on the electromagnetic signal radiated by the transmitter antenna apart from the direct line of sight wave. Reflection of the radiated wave leads to components of it traveling through multiple paths to get to the receiving antenna. Each of these reflected wave components arriving at the receiving antenna have different amplitudes, phases and arrive at the receiving antenna at different times. It is such that wave components which arrive in phase with the direct line-of-sight signal at the receiver antenna have their amplitudes added-up constructively thereby increasing the strength of the direct signal. While reflected waves that arrive out-of-phase with the direct wave have their amplitudes subtract from that of the direct wave and tend to cancel out the direct signal. In between these ends there exist varying resultant amplitude levels and phases leading to diverse fading or losses in the received signal power.

Particularly for a situation whereby either or both the transmitter and receiver is mobile with the multipath changing with changing reflecting surfaces, the reflected signal components vary so much that the amplitude of the received power is not steady as shown in figure 5 [14].

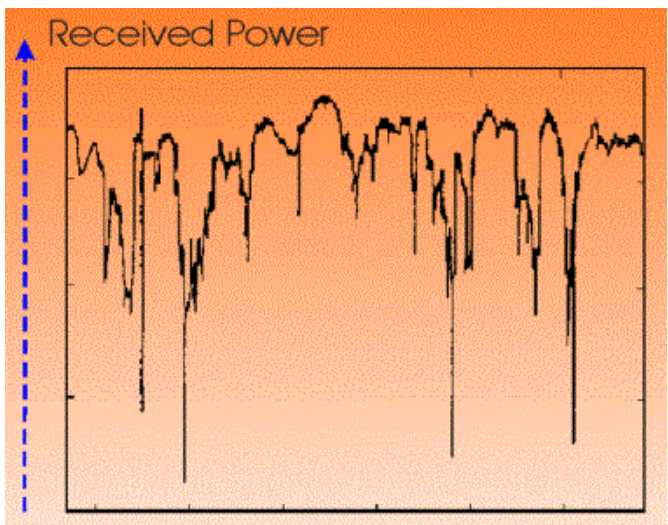

Figure 5: Received Signal Power with varying Multipath conditions.

Signals propagation under multipath phenomenon suffer interference that brings down the signal to noise ratio (SNR) and the bit error rates for digital signals. The following are some effects of multipath reception [15]:

- $\quad$ for a fast moving user: rapid fluctuations of the signal amplitude and phase

- $\quad$ for a wideband (digital) signal: dispersion and inter-symbol interference

- $\quad$ for an analog television signal: "ghost" images (shifted slightly to the right)

- $\quad$ for a multicarrier signal: different attenuation at different (sub-)carriers and at different locations

- for a stationary user of a narrowband system: good reception at some locations and frequencies; poor reception at other locations and frequencies

- $\quad$ for a satellite positioning system: strong delayed reflections may cause a severe miscalculation of the distance between user and satellite. This can result in a wrong "fix"

From reflection on smooth surface, for example smooth earth surface, shown in figure, obtaining the distance, $\mathrm{d}$, geometrically, the direct signal distance and the reflected signal distance are given by:

$$
\begin{aligned}
& d_{\text {direct }}=\left[d^{2}+\left(h_{t x}-h_{r x}\right)^{2}\right]^{1 / 2} \\
& d_{\text {reflected }}=\left[d^{2}+\left(h_{t x}+h_{r x}\right)^{2}\right]^{1 / 2}
\end{aligned}
$$

Given that $\mathrm{d}>\mathrm{h}$, it can be noted that [16]:

$$
\Delta \mathrm{d}=\frac{2 \mathrm{~h}_{\mathrm{tx}} \mathrm{h}_{\mathrm{rx}}}{\mathrm{d}}
$$

and, $\quad \Delta \varphi=\frac{4 \pi \mathrm{h}_{\mathrm{tx}} \mathrm{h}_{\mathrm{rx}}}{\mathrm{d} \lambda}$

where $\Delta \varphi$ is the change in phase of the reflected signal from the direct signal. 


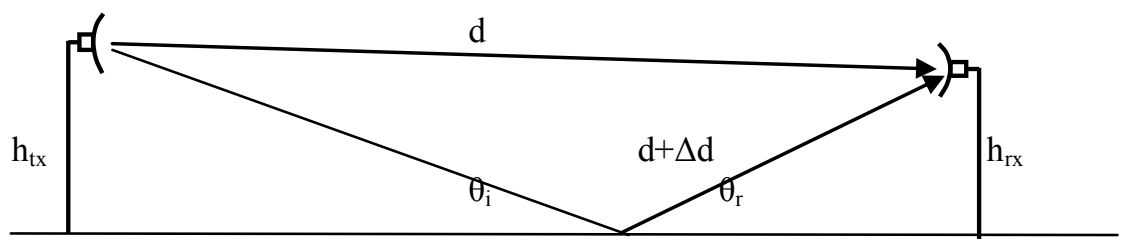

Figure 6: Reflection on a Smooth Surface.

The reflection coefficient $\rho$, usually determines the phase and amplitude of the reflected wave. The value of the reflection coefficient is obtained different depending on which one of $\mathrm{E}$ plane or $\mathrm{H}$ plane that is parallel to the reflecting surface, and the equations are given by [16]:

$\rho_{\mathrm{E}}=\frac{\sin \theta-\sqrt{\left(\epsilon / \epsilon_{0}-\mathrm{j} \sigma / \omega \epsilon_{0}\right)-\cos ^{2} \theta}}{\sin \theta+\sqrt{\left(\epsilon / \epsilon_{0}-\mathrm{j} \sigma / \omega \epsilon_{0}\right)-\cos ^{2} \theta}}$

$\rho_{\mathrm{H}}=\frac{\left(\epsilon / \epsilon_{0}-\mathrm{j} \sigma / \omega \epsilon_{0}\right) \sin \theta-\sqrt{\left(\epsilon / \epsilon_{0}-\mathrm{j} \sigma / \omega \epsilon_{0}\right)-\cos ^{2} \theta}}{\left(\epsilon / \epsilon_{0}-\mathrm{j} \sigma / \omega \epsilon_{0}\right) \sin \theta+\sqrt{\left(\epsilon / \epsilon_{0}-\mathrm{j} \sigma / \omega \epsilon_{0}\right)-\cos ^{2} \theta}}$

where $\varepsilon=$ permittivity and $\sigma=$ conductivity.

Estimating the cumulative multipath loss in link is very unpredictable. There are the ground reflection path loss and the very unpredictable multipath loss due to diverse obstacles and reflectors in-between the transmitting and the receiving antennas. Diffuse reflections occur where surfaces on which the transmitted wave impinges is rough, like rough ground surfaces, as shown in figure 7. This further complicates the predictability of the multipath loss.

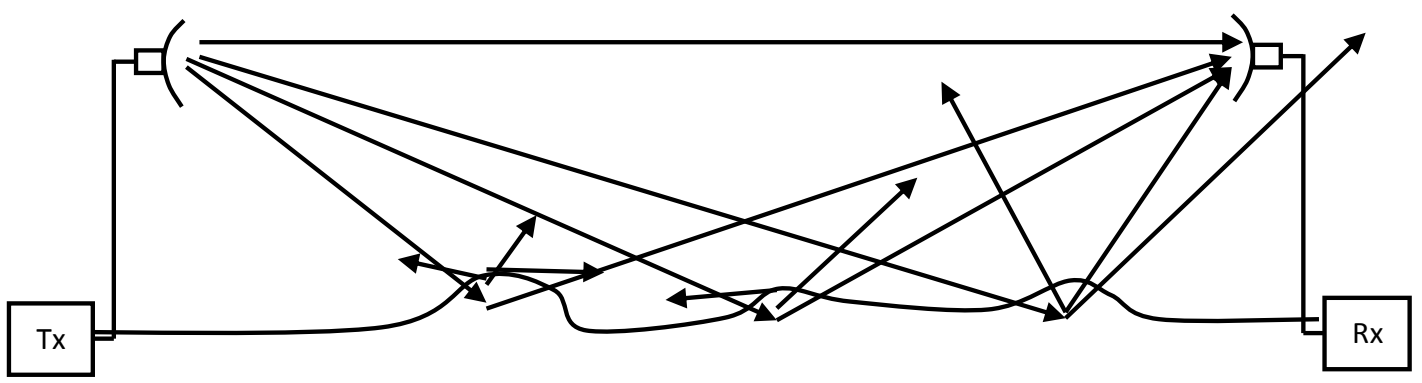

Figure 7: Diffuse Reflections from Rough Ground Surface.

[5] claimed that multipath fading can result in over $30 \mathrm{~dB}$ loss in the transmitted signal and recommended that adequate link margin must be factored into the link budget during link design in order to overcome this loss. Otherwise, the reliability of the wireless link will be seriously hampered. This extra RF power radiated to overcome multipath fading is known as the fade margin and the amount of it factored into the design depends on the level of reliability desired for the link. Some other techniques for mitigating multipath fading include antenna diversity techniques, adaptive channel equalization, and spread spectrum systems [17]

\subsection{Obstruction losses}

The Fresnel Zone is an elliptical volume around the line of sight which must be clear of any obstruction for maximum power to reach the receiver's antenna. The presence of obstructions like trees, tall buildings, hilltops and so on, within the Fresnel Zone reduces the integrity of effectiveness of the propagation link. Calculating power losses due to obstructions within the link could be very complex and unpredictable, because various shapes of obstructions could be found within various links. And, in a particular link the new obstructions could spring up at any time. Therefore, to reduce the complexity of link budget calculations, only line of sight with sufficient Fresnel Zone clearance are considered.

\subsection{Diffraction losses}

Diffraction loss occurs either when the radio wave between the transmitter and receiver is obstructed by a surface or an object that has sharp edges or as it travels around the curvature of the earth surface. Depending on the type of terrain and vegetation on the radio path, the sharp edge obstruction poses minimum 
diffraction loss, while smooth earth curvature poses maximum diffraction loss. These are two idealized obstacle models which represent extreme and opposite conditions which are references for obtaining a theoretical evaluation of diffraction loss in radio propagation [14]. For practical diffraction loss calculation over average terrain formula is given by [7]:

$\mathrm{A}_{\mathrm{d}}=-20 \mathrm{~h} / \mathrm{F}_{1}+10(\mathrm{~dB})$

where $\mathrm{h}$ is the height difference $(\mathrm{m})$ between most significant path obstacle and the path trajectory $(\mathrm{h}$ is negative if the top of the obstruction of interest is above the virtual line-of-sight),

$F_{1}$ is the radius of the first Fresnel ellipsoid given by:

$\mathrm{F}_{1}=17.3 \sqrt{\frac{\mathrm{d}_{1} \mathrm{~d}_{2}}{\mathrm{fd}}}(\mathrm{m})$

where: $f$ is frequency $(\mathrm{GHz}), \mathrm{d}$ is path length $(\mathrm{km})$, and $\mathrm{d}_{1}$ and $\mathrm{d}_{2}$ are distances in $\mathrm{km}$ from the terminals to the path obstruction.

\section{Accounting for Power Losses in Link Budgeting}

These losses are time variants, the best way to account for them in link budget calculations is to assume the worst case whereby the signal to noise ratio is at its highest degradation or the bit error rate is at its highest value.

In relations to the Equivalent Isotropic Radiated Power (EIRP), the received power calculated in link budgeting is obtained from the Link-Power Budget Formula for the received power $\mathrm{P}_{\mathrm{Rx}}$ given by:

$\mathrm{P}_{\mathrm{Rx}}=[\mathrm{EIRP}]+\mathrm{G}_{\mathrm{Rx}}-[$ LOSSES $] \quad(\mathrm{dB})$

where the equivalent isotropic radiated power is $[\mathrm{EIRP}]=\mathrm{P}_{\mathrm{Tx}}+\mathrm{G}_{\mathrm{Tx}} \quad(\mathrm{dBW})$

$\mathrm{P}_{\mathrm{Tx}}$ is the transmitted power in $\mathrm{dBW}, \mathrm{G}_{\mathrm{Tx}}$ is the transmitter antenna gain in $\mathrm{dB}$ and $\mathbf{G}_{\mathbf{R x}}$ is the receiver antenna gain in $\mathrm{dB}$.

The aggregated losses in the link comprises of all losses at the transmitter and receiver ends and in the medium, plus attenuations suffered by the transmitted signal due to the effects of multipath fading, reflection, diffraction and scattering. These are captured as follows:

$[\mathrm{LOSSES}]=\mathrm{L}_{\mathrm{FSPL}}+\mathrm{L}_{\mathrm{TXFL}}+\mathrm{L}_{\mathrm{RXFL}}+\mathrm{L}_{\mathrm{TAML}}+\mathrm{L}_{\mathrm{RAML}}+\mathrm{L}_{\mathrm{PML}}+\mathrm{L}_{\mathrm{OPL}}$

where:

$\mathrm{L}_{\mathrm{FSPL}}=$ free-space path loss in $\mathrm{dB}=\mathrm{P}_{\mathrm{T}} / \mathrm{P}_{\mathrm{R}}$ (in watts)

$\mathrm{L}_{\mathrm{TXFL}} \& \mathrm{~L}_{\mathrm{RXFL}}=$ transmitter and receiver feeder loss in $\mathrm{dB}$

$\mathrm{L}_{\mathrm{TAML}} \& \mathrm{~L}_{\mathrm{RAML}}=$ transmitter and receiver antenna mismatch loss in $\mathrm{dB}$

$\mathrm{L}_{\mathrm{PML}}=$ polarization mismatch loss in $\mathrm{dB}$.

$\mathrm{L}_{\mathrm{OPL}}=$ all other losses on the transmission path, which comprised of obstruction losses, diffraction losses, atmospheric absorption loss and attenuation due to multipath fading, in $\mathrm{dB}$.

\section{Conclusions}

This work endeavoured to aggregate all the feasible losses and attenuations suffered by a transmitted signal in a terrestrial radio link. Consideration was not particularly given to satellite radio link, which would involve some more complex atmospheric absorption by water vapours and atmospheric gases, atmospheric attenuation due rain, hail, fog, and snow, and ionospheric disturbances and cosmic radiations. Also this work did not give consideration to system effects like System Noise because the effects of losses within the link coverage is assumed to be from the output of the transmitter power amplifier circuitry and the input to the receiver RF Amplifier.

\section{References}

[1]. Racom, Ray - Microwave Link, Link Calculation http://www.racom.eu/eng/products/m/ray/calcul.html

[2]. Ian P., Link Budget, http://www.radio-electronics.com/info/propagation/path-loss/link-budget-calculation-formula-equation.php

[3]. Stoehr M. (2011), Radio Link-Budget Calculations for ISM-RF Products, Mxim Integrated, http://www.maximintegrated.com/an5142

[4]. Shamanna P. (2013), "Simple Link Budget Estimation and Performance Measurements of Microchip 2.4 GHz Radio Modules", Microchip Technology Inc.,

[5]. Tranzeo, Wireless Link Budget Analysis - How to Calculate Budget for your Wireless Network, Tranzeo Wireless Technologies Inc., Pitt Meadow, BC, Canada. 
[6]. Stevens B. (1998), “Antenna Properties and their impact on Wireless System Performance”, Cushcraft Corporation 48 Perimeter Road, Manchester, NH 03013

[7]. ITU-R P.530 -15 (2013), "Propagation data and prediction methods required for the design of terrestrial line-of-sight systems"

[8]. Wang Tao, Long Term Evolution (LTE) Radio Access Network Planning Guide, Huawei LTE Planning, Huawei Technologies Co. Ltd., Shenzhen, China, 2011

[9]. Harry Lythall (SM0PVO), RF Feeders, http://www.sm0vpo.com/antennas/feeder0.htm

[10]. Donjiang Qiao

[11]. Nikolova, N. K. (2007), Polarization and Related Antenna Parameters, ANTENTOP - 01 - 2007, \#009, www.antentop.org

[12]. NAWCWPNS TP8347 (1999), Electronic Warfare and Radar Systems Engineering Handbook, Naval Air Systems Command, Avionics Department AIR-4.5 Washington, DC 20361.

[13]. Durgin, G. and Rappaport T. S. (1998), Measurements and Models for Radio Path Loss and Penetration Loss In and Around Homes and Trees at $5.85 \mathrm{GHz}$, IEEE Transactions on Communications, Vol. 46, No. 11, November 1998.

[14]. Moreno Luigi (2010), Point - to - Point Radio Link Engineering, A Self-Learning E-Book Based Course, By Radio Engineering Services mailto:1.moreno@radioengineering.it.

[15]. Linmartz Jean-Paul M. G., (1996), Wireless Communication, The Interactive Multimedia CD-ROM, Baltzer Science Publishers, P. O. Box 37208, 1030 AE Amsterdam, ISSN 1383 4231, Vol. 1 (1996), No.1

[16]. Willis M. (2007), An Introduction to Radiowave propagation, http://www.mike-willis.com/Tutorial/PF16.htm

[17]. Zyren J. and Petrick A., (1998) Tutorial on Basic Link Budget Analysis, Intersil Corporation, http://www.intersil.com 